\title{
Delay in diagnosis and treatment of patients with cases of imported malaria in Poland - one center's experience
}

\author{
JACEK CZEPIEL ${ }^{1, A-F}$, SARAH GOLDMAN², в, E, F, ANASTAZJA SZLAUER-STEFAŃSKA2, в, E, , , \\ ALEKSANDRA MIELIMONKA ${ }^{2, \mathrm{~B}, \mathrm{E}, \mathrm{F}}$, GRAŻYNA BIESIADA ${ }^{1, \mathrm{D}-\mathrm{F}}$, ANNA KALINOWSKA-NOWAK ${ }^{1, \mathrm{E}}$, \\ ALEKSANDRA WESEŁUCHA-BIRCZYŃSKA ${ }^{3, \mathrm{E}}$, WILLIAM PERUCKI ${ }^{4, \mathrm{E}}$, ALEKSANDER GARLICKI $^{1, \mathrm{D}, \mathrm{E}}$
}

\author{
${ }^{1}$ Department of Infectious and Tropical Diseases Chair of Gastroenterology, Hepatology and Infectious Diseases, \\ Jagiellonian University Medical College, Cracow, Poland \\ ${ }^{2}$ Student's Scientific Society, Jagiellonian University, Medical College, Cracow, Poland \\ ${ }^{3}$ Department of Chemical Physics, Faculty of Chemistry, Jagiellonian University, Cracow, Poland \\ ${ }^{4}$ Department of Medicine, John Dempsey Hospital, University of Connecticut, Farmington, CT, USA
}

A - Study Design, B - Data Collection, C - Statistical Analysis, D - Data Interpretation, E - Manuscript Preparation, F - Literature Search, G - Funds Collection

Summary Background. Increasingly, Polish citizens are traveling to malaria endemic regions; thus, physicians, especially primary care physicians, should be educated to recognize and treat malaria. Diagnosis and treatment of malaria encounters many difficulties in Poland.

Objectives. The aim of the study was to analyze malaria chemoprophylaxis, the time from first symptoms to hospitalization and the process of diagnosis and treatment of patients with malaria.

Material and methods. The medical records of patients diagnosed with malaria, hospitalized between 2012 and 2016 in the Department of Infectious Diseases of the University Hospital, Cracow, Poland, were analyzed.

Results. 37 subjects with a median age of 32 years (interquartile range IQR: 28-40), mostly returning from Africa $(78 \%, n=29)$, were studied. Proper chemoprophylaxis was used in 6 cases (16\%). The median length of stay in malaria endemic countries was one month. Plasmodium falciparum was the most frequent species (74\%). The mean time to treatment after symptom onset was 5 days (range: 1-27 days).

Conclusions. The clinical presentation of malaria in the study group was usually typical. Diagnostic delay resulted from not taking malaria into consideration during the initial differential diagnosis of fever. Few travelers use chemoprophylaxis, hence the awareness of malaria in individuals who have traveled to endemic zones should be enhanced. In a patient presenting with fever, malaria should always be considered in a differential diagnosis if there is a history of travel to a malaria-endemic zone.

Key words: chemoprophylaxis, diagnosis, malaria, treatment, Plasmodium.

Czepiel J, Goldman S, Szlauer-Stefańska A, Mielimonka A, Biesiada G, Kalinowska-Nowak A, Wesełucha-Birczyńska A, Perucki W, Garlicki A. Delay in diagnosis and treatment of patients with cases of imported malaria in Poland - one center's experience. Fam Med Prim Care Rev 2017; 19(2): 93-97, doi: https://doi.org/10.5114/fmpcr.2017.67859.

\section{Background}

Malaria is one of the most important human parasitic diseases and is the leading cause of deaths in tropical zones. It is caused by parasites of the Plasmodium genus, among which 5 species are pathogenic. The majority of malaria cases are caused by $P$. falciparum and $P$. vivax, whereas $P$. ovale, $P$. malariae and zoonotic $P$. knowlesi are less frequent. $P$. falciparum causes the most severe form of malaria, along with the majority of deaths from malaria. The disease is most commonly transmitted by an infected female Anopheles mosquito [1]. There are 400 Anopheles species in the world, but only 30 are malaria vectors [2].

According to the World Health Organization's (WHO) World Malaria Report 2015, transmission risk exists in 95 countries, mostly in Africa, Asia, South America and some Pacific islands. Almost 3.2 billion people inhabit these areas, and almost 1 billion people live in high transmission risk areas (> 1 case per 1000 population). Malaria primarily affects low income countries, which complicates efforts to combat the infection. Global financing for malaria control reached 2.5 billion USD in 2014. To achieve a $75 \%$ reduction in malaria incidence and deaths, an- nual financing should be increased to 7.7 billion USD by 2025. Although the number of malaria cases has fallen since 2000, the incidence rate is still high (214 million cases in 2015). 70\% of deaths from malaria occurred in children under 5 years of age. $80 \%$ of deaths in 2015 were in the African Region, mostly in SubSaharan Africa. $P$. vivax is estimated to have been responsible for $\mathbf{1 3 . 8}$ million cases of malaria globally in 2015 and accounted for approximately half the total number of cases of malaria outside Africa. P. falciparum is the most prevalent malaria parasite on the African continent. It is responsible for most malaria-related deaths globally. The exact percentage of the remaining three species is difficult to determine; however, they cause malaria much less frequently than $P$. vivax/P. falciparum [3].

Poland was a malaria-endemic country until the 1950s, with endemic transmission of $P$. vivax [4]. The highest rate of incidence was noted in the 1920s, with rates of up to 197.1/100 000 inhabitants [5]. Although Poland was declared as a malaria-free country in 1968, there is still a possibility of local transmission of the Plasmodium genus. There are 5 species of Anopheles mosquitos in Poland, all potentially able to infect themselves from the blood of a patient with parasitemia. In recent years, only imported cases of malaria have been diagnosed with a predom- 
inance of $P$. falciparum malaria [6, 7]. The rate of death from severe malaria in Poland in recent years has been around $1.4 \%$ and is 7-16 times higher than in other European countries [5]. Since 1975, all of Europe is malaria-free, although local transmission of malaria in Greece was reported in 2010-2012. This suggests that sporadic Plasmodium transmission in the Mediterranean Basin is still possible [6].

\section{Objectives}

The aim of the study was to analyze malaria chemoprophylaxis, the time from first symptoms to hospitalization and the process of diagnosis and treatment among patients with malaria.

\section{Material and methods}

We planned a retrospective case review study in which we included patients diagnosed with malaria between 2012-2016 at the Department of Infectious Diseases of University Hospital in Cracow. The diagnosis of malaria was made based on medical history, laboratory tests, as well as malaria rapid diagnostic tests, and was confirmed by microscopic examination of peripheral blood smears. We used the OptiMAL-IT Rapid Malaria Test Kit (Bio-Rad, France). Thin blood smears were prepared as typical for routine hematologic staining. The slides were dried and fixed with methanol for 30 seconds and then stained with a Giemsa solution for the next 30 minutes. The dried slides were viewed at $1000 \mathrm{X}$ magnification with oil immersion. Thick blood smears were performed using about $5 \mu \mathrm{l}$ of blood; the drop was put in the center of a slide and spread to cover an area of approximately $10-15 \mathrm{~mm}$ diameter. The slide was then put into buffered water to allow cell lysis until the hemoglobin did not fall away from the smear, and then the excess water was removed. After that, the slides were stained with a Giemsa solution for 30 minutes, rinsed with tap water and dried. Thick smears were examined under microscope as described above [7]. Blood parameters were measured using routine techniques. We analyzed data regarding time, travel destination, occupation, use of malaria prophylaxis, blood tests which were the most important in malaria diagnosis, such as quick rapid test for malaria detection, microscope blood smear assessment, white blood cells (WBC), red blood cells (RBC), hemoglobin, hematocrit, platelets, C-reactive protein (CRP), alanine transaminase (ALT), bilirubin, creatinine.

The data was statistically analyzed. Normal distribution of variables was checked using the Shapiro-Wilk test. The results were presented as arithmetic means and their standard deviations or medians with interquartile ranges. Statistical analysis was performed using Statistica 12 software (StatSoft Inc., USA).

\section{Results}

During the study period (years 2012-2016), 37 subjects were hospitalized. The median age was 32 years (interquartile range, IQR: $28-40) .25$ patients were men (68\%), 12 were women (32\%). 15 patients (41\%), including missionaries/engineers, worked in malaria-endemic countries. There was also 1 case of a laboratory technician who worked on Ebola virus detection in Sierra Leone during the period of that epidemic. 9 patients (24\%) were travelling as tourists. $4(11 \%)$ patients studied or worked in Poland, but originally came from malaria-endemic countries. In 9 (24\%) cases, the purpose of travel was unknown.

Patients with malaria returned from Africa in 29 cases (78\%), from Asia in 6 cases $(16 \%)$, and $1(3 \%)$ returned from Central America. The country of travel in 1 case (3\%) was unknown.

The most visited countries in Africa were Kenya and Uganda (5 patients each), Cameroon and Congo (4 patients each). In- dia ( 2 patients) was the most visited country in Asia. 1 patient returned from the Dominican Republic in Central America. 33 patients (89\%) were Polish, 1 patient was Kenyan, 1 was South Sudanese, 1 was from Congo, and 1 was Nigerian. Among the 33 Poles, 1 person was a permanent resident of Uganda.

We asked patients about chemoprophylaxis, and we assessed the answer as "proper" when the patients took $100 \%$ of the pills prescribed by a physician. Proper chemoprophylaxis was followed in only 6 cases $(16 \%), 5$ patients $(14 \%)$ followed chemoprophylaxis irregularly, 24 (65\%) did not follow chemoprophylaxis at all. We were not able to determine chemoprophylaxis in 2 cases (5\%). Among 5 patients who followed chemoprophylaxis irregularly, 3 persons used atovaquon/proguanil, 1 mefloquine, and 1 doxycycline. The median length of stay in malaria endemic countries was 1 month. The mean time to treatment after symptom onset was 5 days (range: 1-27 days). Table 1 presents the statistics of laboratory variables checked upon admission.

\begin{tabular}{|l|l|l|l|l|}
\hline \multicolumn{5}{|c|}{ Table 1. Laboratory parameters checked upon admission } \\
\hline Parameter & $n$ & median & Q25 & Q75 \\
\hline WBC $\left(\times 10^{3} / \mathrm{I}\right)$ & 37 & 4.7 & 3.5 & 6.1 \\
\hline RBC $\left(\times 10^{6} / \mathrm{I}\right)$ & 37 & 4.5 & 4.3 & 4.9 \\
\hline Hemoglobin $(\mathrm{g} / \mathrm{dl})$ & 37 & 13.3 & 12.1 & 14.3 \\
\hline Hematocrit $(\%)$ & 37 & 39.0 & 36.0 & 41.0 \\
\hline Platelets $\left(\times 10^{3} / \mathrm{I}\right)$ & 37 & 82 & 63 & 137 \\
\hline CRP $(\mathrm{mg} / \mathrm{I})$ & 32 & 68.1 & 30.3 & 124.2 \\
\hline ALT $(\mathrm{U} / \mathrm{I})$ & 34 & 49 & 32 & 81 \\
\hline Bilirubin $(\mu \mathrm{mol} / \mathrm{I})$ & 35 & 21.2 & 12.1 & 36.0 \\
\hline Creatinine $(\mu \mathrm{mol} / \mathrm{I})$ & 36 & 79 & 61 & 89 \\
\hline
\end{tabular}

ALT - alanine transaminase, CRP - C-reactive protein, RBC - red blood cells, Q25 - first quartile, Q75 - third quartile, WBC - white blood cells.

Median platelet count was lower than the reference range, and median CRP, ALT and bilirubin was above the reference range. Other variables were in the reference range.

We also checked the percentage of patients diagnosed with malaria who had laboratory and radiological parameters outside normal ranges (Table 2).

\begin{tabular}{|c|c|c|}
\hline Parameter & $n$ & $\%$ \\
\hline WBC $\left(\times 10^{3} / I\right)$ & 14 & 38 \\
\hline $\operatorname{RBC}\left(\times 10^{6} / \mathrm{I}\right)$ & 5 & 14 \\
\hline Hemoglobin (g/dl) & 3 & 8 \\
\hline Hematocrit (\%) & 14 & 38 \\
\hline Platelets $\left(\times 10^{3} / \mathrm{I}\right)$ & 26 & 70 \\
\hline $\mathrm{CRP}(\mathrm{mg} / \mathrm{l})$ & 27 & 84 \\
\hline $\operatorname{ALT}(\mathrm{U} / \mathrm{I})$ & 25 & 74 \\
\hline Bilirubin $(\mu \mathrm{mol} / \mathrm{l})$ & 18 & 51 \\
\hline Creatinine $(\mu \mathrm{mol} / \mathrm{l})$ & 1 & 3 \\
\hline Hepatomegaly & 6 & 19 \\
\hline Splenomegaly & 20 & 54 \\
\hline
\end{tabular}

ALT - alanine transaminase, CRP - C-reactive protein, RBC - red blood cells, WBC - white blood cells.

CRP, although a non-specific parameter, was most frequently out of the reference range. Malaria specific values (low hematocrit, low platelet count, high ALT, bilirubin, splenomegaly) were present in most cases. Only 2 patients in the studied group had all parameters within the reference range. 
A malaria rapid diagnostic test was performed in 32 patients. In 28 cases, the test was positive (88\%), and in 4 patients, it was negative $(12 \%)$.

All 37 cases of malaria were confirmed by microscopic examination of blood film. In 6 patients, Plasmodium was present, but the species of parasite was not identified, because some patients were already self-treated (a missionary working in the tropics). Among 31 patients with identified species of Plasmodium, Plasmodium falciparum was the most frequent species ( $n=23,74 \%)$; in 7 cases (23\%), P. vivax was the pathogen; in 3 cases $(10 \%), P$. ovale was the pathogenic species. The total sum exceeds $100 \%$, because 2 patients had mixed infections (P. falciparum-P. ovale or $P$. vivax-P. ovale). Median parasitemia at admission was $1.4 \%$ (standard deviation, $\mathrm{SD} \pm 2.9$ ).

In the study group, the most commonly used antimalarial drugs were artemisinin derivatives (artesunate or artemether with lumefantrine, 21 patients), atovaquone/proguanil (12 patients), chloroquine ( 3 patients) and quinine with doxycycline (1 patient). Following basic treatment, primaquine was used in patients infected with $P$. vivax or $P$. ovale.

\section{Discussion}

Malaria is an important tropical disease imported into the Małopolska province in Southern Poland. Its presence is likely related to tourism; however, it is mostly associated with work in endemic areas, which was proven in a recent statistical analysis that covered all of Poland $[8,9]$. P. falciparum and $P$. vivax are responsible for the majority of cases of malaria. Our study has shown that the majority of infections were caused by $P$. falciparum or P. vivax. As many as 30 out of 31 patients (97\%), in whom the blood smear confirmed the type of Plasmodium, were infected by these species. This is in line with global epidemiological trends. The majority of cases were associated with travel to Africa (78\%). In the Małopolska province, there are two large Catholic dioceses (Cracow and Tarnow), where missionary activity is of great importance. It is thus not surprising that many malaria patients were either clergy or laymen involved in missionary work or family members visiting relatives on such missionary work. This explains the predominance of malaria originating from African countries. It should also be noted that in recent years, the number of people working in Africa or traveling to Asia has increased. The length of their stay varied due to the reason for traveling - up to 1 month for tourists or over 1 year for professionals. The subjects mostly visited countries in the eastern part of Sub-Saharan Africa, such as Uganda, Kenya and Tanzania. Among non-African countries, the most common were India and Indonesia.

The mean incubation period, as of the moment of mosquito bite, ranges from 10 to 14 days and depends on Plasmodium species type. Due to production of intrahepatic forms by $P$. vivax and $P$. ovale, malaria symptoms may appear even 1 year after the bite. Early manifestations are non-specific and predominantly include malaise, headache, muscle pain, abdominal discomfort and fatigue. Fever, nausea and vomiting appear subsequently. Additionally, hepatosplenomegaly and jaundice may occur. Having analyzed our data, we need to emphasize that there is an issue with the prompt recognition of malaria patients. The mean time-to-admission to our department was 5 days; however, the longest period was 27 days. In the analyzed group, no fatal cases were observed, and delayed diagnosis was reported in patients with a less severe form of malaria caused by $P$. vivax or $P$. ovale. A delay of several days in the start of $P$. falciparum malaria treatment may lead to the patient's death. Patients, in general, willingly mention travel to tropical countries during the taking of a history, and even if they did not, it still should be a routine question asked by each physician when looking for the cause of fever. Our results show that patients visiting Emergency Departments or Family Practices have the same typical symptoms and laboratory results. A patient who has come back from malaria endemic regions with fever, anemia, thrombocytopenia, increased alanine transaminase and bilirubin levels, as well as splenomegaly, should not cause any diagnostic problems.

If malaria is suspected, it is possible to do a rapid immunochromatographic test to detect the Plasmodium antigen. There are different tests available that have high sensitivity and specificity, especially towards P. falciparum [10]. They provide quick results and may be used by untrained personnel; however, they do not reflect the level of parasitemia, which is crucial for patient prognosis and making the right therapeutic decision. Therefore, a rapid test result must be confirmed using another method [11]. The gold standard for malaria diagnosis remains microscopic examination of a blood drop drawn from the fingertip, by means of a thick and thin blood smear [11]. A thick blood smear allows Plasmodium detection even at low parasitemia levels, while a thin blood smear is used for parasite species differentiation. If malaria is suspected, a microscopic exam should be performed at least 3 times and must be repeated during treatment to monitor the response to therapy [5]. Assessment of the serum antibody level using indirect immunofluorescence is essential for retrospective confirmation of malaria. It has low sensitivity, however, and it does not allow one to distinguish between active and past infection or between different Plasmodium species [5]. Polymerase chain reaction (PCR) analysis plays a vital role in patients with mixed infection, low parasitemia or inconsistent results [12]. New diagnostic techniques using genome sequencing might also be applicable in parasite drug resistance [13]. The usefulness of rapid tests in the diagnosis of malaria has been confirmed in our study. We did not observe any positive malaria tests and negative microscopy results from the same patient during our study period. All false negative results (i.e. a negative malaria test and a positive microscopy test) were found in patients with low parasitemia, which is why, as previously mentioned, a blood smear should also always be performed. It is worth emphasizing that patients with low parasitemia generally have a better prognosis.

Anti-malarial prophylaxis consists of protection against mosquito bites and chemoprophylaxis. The efficacy of chemoprophylaxis was described for the first time in 1901 by Giovanni Battista Grassi, who fought the disease in Agro Pontino, a region in central Italy. We use chloroquine (in regions where $P$. falciparum resistance to antimalarial drugs does not occur), as well as atovaquon/proguanil, doxycycline, mefloquine and primaquine (if there is resistance), as malaria chemoprophylaxis. Chemoprophylaxis should only be recommended by physicians with sufficient knowledge and experience, and it ought to be based on information regarding the area to be visited, time of travel and health status of the traveler [14]. In our study group, only a few patients followed chemoprophylaxis properly, $65 \%$ of patients did not use any antimalarial drugs and some (14\%) claimed either irregular use or use only during the initial part of the stay. It is worth mentioning that this proportion is most certainly underrepresented. Among the subjects who claimed to have followed chemoprophylaxis, irrespective of regularity, only one patient was a tourist, while the rest worked in endemic areas. The majority of subjects revealed that it was the employer who provided free antimalarial prophylaxis; hence, it is possible that they did not want to officially admit to exposing their companies to additional costs by not using prophylaxis appropriately. It seems, however, that lack of prophylaxis in patients who traveled for tourist purposes was the result of their low awareness of the danger of malaria. Their arguments, such as price of medication, fear of side effects or poor access to infectious disease specialists, are not justified, as chemoprophylaxis costs are low compared to the costs of travel and potential financial losses resulting from the disease. Moreover, the side effects of the drug are hardly ever observed, and specialist consultation is available in large cities and sometimes even small towns. 
Irrespective of the efficacy of chemoprophylaxis, a number of opinions pertaining its use has recently been published, and recommendations differ across countries and organizations [14]. While the WHO, Centers for Disease Control and Prevention (CDC) and the French Haut Conseil de la Santé Publique France guidelines recommend chemoprophylaxis for most travelers heading to endemic areas $[2,15,16]$, other countries (Germany, Switzerland, Austria) prefer the use of prophylaxis only in high risk regions, such as continental Africa, some parts of Oceania or selected areas of the Amazon Basin [17]. The remaining guidelines contain a mix of the aforementioned recommendations [14]. Data gained by the TropNet expert panel (www. tropnet.net) demonstrates that chemoprophylaxis is used more often in Northern European countries than in the central part of the continent; furthermore, in almost every European country, chemoprophylaxis is used less often than the local guidelines recommend [18].

The current treatment of malaria according to 2016 WHO guidelines is based on the identified parasite species and the severity of the disease [2]. Therapy should be introduced as early as possible, preferably within 48 hours from symptom onset. If the patient followed chemoprohylaxis, a different drug should be chosen for the therapy. $P$. vivax infection acquired in an area where no chloroquine resistance occurs should be treated with this drug. In all other cases, as well as in a mild $P$. falciparum infection, combination therapy should be used. According to WHO guidelines, artemisinin derivatives (e.g.: artemether, artesunate) are recommended as first-line treatment together with lumefantrine, mefloquine or sulfadoxine/pyrimethamine. In $P$. vivax or $P$. ovale infections, the basic treatment should be additionally followed by primaquine use in order to clear the remaining hypnozoites, which are responsible for malaria relapse. In severe forms of the disease, intravenous artesunate is considered as primary therapy. The second-line treatment consists of quinine along with doxycycline administered intravenously. Should malaria occur during pregnancy, the treatment of choice for uncomplicated $P$. falciparum infection remains quinine along with clindamycin in the first trimester, while a combination of artemisinin derivatives is used in both the second and third trimester. In case of $P$. vivax, $P$. ovale, $P$. malariae or $P$. knowlesi infection in the first trimester of pregnancy, quinine or chloroquine is used. Severe malaria in pregnant women in all trimesters is treated with intravenous artesunate. The treatment of children remains similar to that of adults; however, weight-adjusted dosing is used. Moreover, primaquine must be avoided during the first 6 months of life, and doxycycline should not be used in patients under the age of 8 [2].

Our patients were treated according to these recommendations. All of the patients survived, though 2 patients required treatment in the intensive care unit. The reason for this was an altered level of consciousness and severe dyspnoea accompanied by ARDS features. The symptoms resolved during treatment, and no subject required intubation.

\section{Conclusion}

The clinical presentation of malaria in the studied group was, in most cases, typical. The observed diagnostic delay resulted from not taking malaria into consideration during the initial differential diagnosis of fever. Few travelers followed chemoprophylaxis; hence, risk awareness in individuals travelling to endemic zones should be enhanced and an information kit about tropical diseases should be introduced to travel agencies. Quick diagnosis and treatment decrease the risk of death due to malaria. In a patient presenting with fever, malaria should always be considered in the differential diagnosis if there is a history of travel to malaria-endemic areas.

Source of funding: This work was funded by the authors' own resources.

Conflict of interest: The authors declare no conflict of interests.

\section{References}

1. White NJ, Pukrittayakamee S, Hien TT, et al. Malaria. Lancet 2014; 383(9918): 723-735.

2. World Health Organization. International travel and health. Geneva: World Health Organization; 2016.

3. World Health Organization. World malaria report 2015. Geneva: World Health Organization; 2015.

4. Dzbenski TH. Epidemiological situation of malaria in Poland - past, present and future. Wiad Parazytol 2008; 54(3): $205-211$ (in Polish).

5. Knap J, Myjak P, eds. Malaria w Polsce i na świecie. Wczoraj i dziś. Bielsko-Biała: alfa-medica press; 2009 (in Polish).

6. Danis K, Baka A, Lenglet A, et al. Autochthonous Plasmodium vivax malaria in Greece, 2011. Euro Surveill 2011; 16(42): 19993.

7. Warhurst DC, Williams JE. Laboratory diagnosis of malaria. J Clin Pathol 1996; 49(7): 533-538.

8. Stępień M. Malaria in Poland in 2011. Prz Epidemiol 2013; 67(2): 273-275, 373-374.

9. Stępień M. Malaria in Poland in 2012. Prz Epidemiol 2014; 68(2): 29-33, 387-388.

10. Abba K, Deeks JJ, Olliaro P, et al. Rapid diagnostic tests for diagnosing uncomplicated $P$. falciparum malaria in endemic countries. Cochrane Database Syst Rev 2011; 7: CD008122.

11. Whitty CJM, Chiodini PL, Lalloo DG. Investigation and treatment of imported malaria in non-endemic countries. BMJ 2013; 346: $\mathrm{f} 2900$.

12. Myjak P, Nahorski W, Pieniazek N, et al. Usefulness of PCR for diagnosis of imported malaria in Poland. Eur J Clin Microbiol Infect Dis 2002; 21(3): 215-218.

13. Robinson T, Campino SG, Auburn S, et al. Drug-resistant genotypes and multi-clonality in Plasmodium falciparum analysed by direct genome sequencing from peripheral blood of malaria patients. PLoS One 2011; 6(8): e23204.

14. Calleri G. Malaria prophylaxis and guidelines. Infection 2014; 42(5): 913-916.

15. Centers for Disease Control and Prevention. The Yellowbook 2016. Health information for international travel. Atlanta: CDC; 2016.

16. Haut Conseil de la Sante' Publique. Recommandations sanitaires pour les voyageurs 2016. Paris: Santé publique France; 2016.

17. Deutsche Gesellschaft fuer Tropenmedizin und Internationale Gesundheit (DTG). Empfehlungen zur Malariavorbeugung 2012. Hamburg: DTG; 2012.

18. Calleri G, Behrens RH, Bisoffi Z, et al. Variability in malaria prophylaxis prescribing across Europe: a Delphi method analysis. J Travel Med 2008; 15(5): 294-301.

Tables: 2

Figures: 0

References: 18 
Address for correspondence:

Jacek Czepiel, MD, PhD

Klinika Chorób Zakaźnych i Tropikalnych UJ CM

ul. Śniadeckich 5

31-531 Kraków

Polska

Tel.: +48 12 424-73-49

E-mail: jacek.czepiel@uj.edu.pl 